\title{
Examination of the Series Analysis Method for Adsorption of a Polymer
}

\author{
Takao ISHINABE \\ Faculty of Engineering, Yamagata University, \\ Yonezawa 992, Japan
}

(Received April 12, 1986)

\begin{abstract}
A series analysis method to estimate the critical point and exponents for adsorption of a polymer was tested using the non-reversal random walk model, for which analytical values are available. The values estimated from the exact enumeration data for up to 18 steps on the tetrahedral lattice are in good agreement with the expected ones for both cases having penetrable and impenetrable interface planes. The result suggests the possibility that for the self-avoiding walk model in penetrable case the estimate of crossover exponent $\varphi$ is different between two methods; one uses the relation $\varphi=1 / q$ ( $q$ : the exponent for the free energy) and the other uses the scaling relation $\varphi=\theta-1(\theta$ : the exponent for the energy).
\end{abstract}

KEY WORDS Adsorption of a Polymer / Series Analysis / Critical Point /

Critical Exponent / Crossover Exponent / Non-Reversal Random Walk /

Adsorption of polymers at surfaces has attracted the attention of a large number of investigators. The treatments based on the random walk $(\mathrm{RW})$ model have been reviewed by Barber and Ninham. ${ }^{1}$ Recently the problem incorporating the excluded volume effect has been treated prevalently by the methods of Monte Carlo and exact enumeration for self-avoiding walks (SAWs) interacting with surfaces. ${ }^{2,3}$ Such a problem is closely related with the surface magnetism for a semi-infinite system of $n$-component spins. ${ }^{4-6}$ The adsorption transition point is essentially equivalent to the multicritical surface-bulk point for the spin system $^{7}$; the critical exponent $q$ for the free energy of an adsorbed chain corresponds to the inverse of a surface crossover exponent $\varphi$, i.e., $q=1 / \varphi(n \rightarrow 0)^{7,8}$

de Gennes ${ }^{4}$ has predicted the relation $\varphi=$ $1-v$ ( $v$, the correlation length exponent) from the scaling argument of polymer adsorption. This relation is equivalent to the Bray and Moore conjecture ${ }^{9}$ for the $O(n)$ model obtained from the renormalization group calculation of $\varphi$ to first order in $\varepsilon$ ( $\varepsilon=4-d ; d$, the spatial dimension). However, Diehl and Dietrich's calculation ${ }^{10}$ to second order in $\varepsilon$ is incompatible with the relation. In previous papers, ${ }^{11}$ we estimated that $q=$ $1.7 \pm 0.3(d=3)$ and $q \simeq 1.9(d=2)$ for the adsorption of a polymer at an impenetrable surface from a series analysis of the exact enumeration data for SAWs. These results are in conflict with the de Gennes-Bray-Moore conjecture since ${ }^{12} v=3 /(d+2)$. Eisenriegler et $a .^{7}$ obtained the value $\varphi=0.59 \pm 0.02$ for $d=3$, which supports our estimate, from the Monte Carlo result for SAWs combined with the concept of critical phenomena. For $d=2$, a real space renormalization group study ${ }^{13}$ has yielded $\varphi=0.55 \pm 0.15$, which also supports our estimate. After consideration of these results, de Gennes and Pincus ${ }^{14}$ has modified the previous scaling theory ${ }^{15}$ introducing the "proximal exponent."

On the other hand, Hammersley et al. ${ }^{16}$ have treated SAWs interacting with a penetrable plane as a model of polymer adsorption 
at a liquid-liquid surface; it is closely connected with the critical behavior of the spin system near a plane of defects. ${ }^{17}$ They have shown rigorously that there exists a transition point in this case as well as in a semi-infinite system. We estimated the same values ${ }^{8}$ of $q$ for $d=2$ and 3 as those for the semi-infinite case from exact enumerations of SAWs. The breakdown of the de Gennes-Bray-Moore conjecture has been suggested also in this case while the conjecture is thought valid in general for such an infinite system. ${ }^{17,18}$ More recently, $\mathrm{Kremer}^{19}$ estimated $\varphi$ for the infinite system which supports the conjectur $\varphi=1-v$ using a Monte Carlo simulation of SAWs on the tetrahedral lattice. Consequently, it seem worthwhile to test the validity of series analysis technique for the adsorption of a polymer chain using a walk such as non-reversal random walk (NRRW) for which rigorous results are known although the validity has been established for the two-dimensional Ising model $^{20}$; NRRW is RW for which direct reversal is not allowed.

\section{ADSORPTION TRANSITION OF A NON-REVERSAL RANDOM WALK CHAIN}

Consider $N$-step NRRWs on a lattice which interact with the interfacial plane either penetrable or impenetrable. Taking into account interaction energy $\varepsilon$ for a vertex in the plane (liquid-liquid or solid-liquid surface), we can write the partition function of such a chain as

$$
Q_{N}(\omega)=\sum_{m=1} C_{N}(m) \mathrm{e}^{m \omega}
$$

where $C_{\mathrm{N}}(m)$ is the number of walks with $m$ vertices in the plane and $\omega=-\varepsilon / k T$. Introducing the reduced free energy

$$
A(\omega)=\lim _{N \rightarrow \infty} N^{-1} \log Q_{N}(\omega)
$$

it is shown that ${ }^{21}$ there exists a critical value, i.e., an adsorption transition point

$$
\omega_{\mathrm{c}}=\sup \left\{\omega: A=A_{0}\right\}
$$

where $A_{0}$ is a constant. For weak adsorption the critical exponent $q$ can be defined by

$$
A-A_{0} \sim\left(\omega-\omega_{\mathrm{c}}\right)^{q}, \omega \rightarrow \omega_{\mathrm{c}}+0
$$

For the infinite system with penetrable plane, we have $\omega_{\mathrm{c}}=0$, i.e., the adsorption transition occurs at an infinitely small monomer-interface interaction as is seen for the SAW model. ${ }^{8,16}$ The value of $\omega_{c}$, however, depends on the type of lattice for the semiinfinite system. Rubin ${ }^{22}$ has shown for the RW model,

$$
\omega_{\mathrm{c}}=\log \frac{2 z}{z+z_{0}}
$$

$z$ being the coordination number of a lattice and $z_{0}$ that of a (two-dimensional) surface lattice. Although a rigorous value of $\omega_{c}$ has not been obtained for the NRRW model, we can get the conjecture of $\omega_{c}$ by the substitution: $z \rightarrow z-1$ and $z_{0} \rightarrow z_{0}-1$ in eq 5. The $\mathrm{RW}$ value $\mathrm{e}^{12,21,22}$ of $q=2$, which is irrespective of $d$, is also valid for NRRWs, in both cases penetrable and impenetrable, since RW and NRRW belong to the same class of universality.

The mean fraction of monomers in an interfacial plane, i.e., the energy of an adsorbed chain per monomer is given by

$$
f_{N}(\omega)=\frac{\sum_{\mathrm{m}} m C_{N}(m) \mathrm{e}^{m \omega}}{N \sum_{m} C_{N}(m) \mathrm{e}^{m \omega}}
$$

and for sufficiently large $N$

$$
f_{N}(\omega) \sim\left\{\begin{array}{l}
N^{-1}, \omega<\omega_{\mathrm{c}} \\
N^{-\theta}, \omega=\omega_{\mathrm{c}} \\
\text { const, } \omega>\omega_{\mathrm{c}}
\end{array}\right.
$$

It is shown that ${ }^{22} \theta=1 / 2$ for the RW model in a semi-infinite case. In general, the relation $\theta=1-\varphi$ holds for NRRWs in both infinite and semi-infinite systems since the scal- 
ing arguments ${ }^{7,8}$ predict $\theta=v$ and $\varphi=1-v$.

\section{EXACT ENUMERATION}

We obtained exact values of $C_{N}(m)$ for $N \leq$ 18 for NRRWs on the tetrahedral lattice in two cases of penetrable and impenetrable interfacial planes. The computations were performed on the ACOS 1000 computer at the Tohoku University. In the infinite system with a penetrable interface, all walks originate in the plane $z=0$ while in the semi-infinite system the walks are restricted to the half-space $z \geq 0$ and have at least one vertex in the plane $z=0$. The interfacial planes ${ }^{11}$ are chosen to contain lattice points in the planer zig-zag conformation such as $\alpha \bar{\beta} \alpha \bar{\beta} \ldots$, where $\alpha=(1,1,1)$ and $\bar{\beta}=(\overline{1}, 1,1)$. The values of $C_{N}(m)$ for semi-infinite system are given in Table I; those for the infinite system are

Table I. Values of $C_{N}(m) / 2$ for the tetrahedral lattice in a semi-infinite system

\begin{tabular}{|c|c|c|c|c|c|c|c|c|c|c|c|c|}
\hline \multirow{2}{*}{$N$} & \multicolumn{12}{|c|}{$m$} \\
\hline & 1 & 2 & \multicolumn{2}{|l|}{3} & 4 & \multicolumn{2}{|l|}{5} & \multicolumn{2}{|l|}{6} & \multicolumn{3}{|c|}{7} \\
\hline 1 & 1 & 1 & & & & & & & & & & \\
\hline 2 & 3 & 2 & & 1 & & & & & & & & \\
\hline 3 & 7 & 8 & & 2 & 1 & & & & & & & \\
\hline 4 & 19 & 21 & & 11 & 2 & & 1 & & & & & \\
\hline 5 & 53 & 63 & & 28 & 15 & & 2 & & 1 & & & \\
\hline 6 & 147 & 192 & & 35 & 40 & & 19 & & 2 & & & 1 \\
\hline 7 & 411 & 578 & 2 & 58 & 133 & & 52 & & 23 & & & 2 \\
\hline 8 & 1163 & 1735 & 7 & 75 & 414 & & 93 & & 64 & & 27 & 7 \\
\hline 9 & 3313 & 5221 & 23 & & 1283 & & 10 & & 69 & & 76 & 6 \\
\hline 10 & 9475 & 15722 & 692 & & 3956 & & 31 & & 70 & & 361 & \\
\hline 11 & 27207 & 47312 & 2072 & & 12125 & & 76 & & 51 & & 1194 & \\
\hline 12 & 78419 & 142325 & 619 & & 37034 & $18 \mathrm{~S}$ & & & 04 & & 4091 & \\
\hline 13 & 226709 & 428127 & $1853^{\circ}$ & & 12871 & 583 & & 29 & & & 13548 & \\
\hline 14 & 657043 & 1287696 & 55440 & & 43328 & 1794 & & 91 & & & 43973 & \\
\hline 15 & 1908459 & 3872322 & 16583 & & 42565 & 549 & & 287 & & & 40898 & \\
\hline 16 & 5554347 & 11642863 & 49609 & & 61798 & 1679 & & 891 & & & 46923 & \\
\hline 17 & 16193409 & 35001981 & 148419 & & 78779 & 5119 & & 2756 & & & 06380 & \\
\hline 18 & 47283843 & 105213762 & 444084 & & 92972 & 15575 & & 8491 & & & 98225 & \\
\hline \multirow{2}{*}{$N$} & \multicolumn{12}{|c|}{$m$} \\
\hline & 8 & 9 & 10 & 11 & 12 & 13 & 14 & 15 & 16 & 17 & 18 & 19 \\
\hline 7 & 1 & & & & & & & & & & & \\
\hline 8 & 2 & 1 & & & & & & & & & & \\
\hline 9 & 31 & 2 & 1 & & & & & & & & & \\
\hline 10 & 88 & 35 & 2 & 1 & & & & & & & & \\
\hline 11 & 469 & 100 & 39 & 2 & 1 & & & & & & & \\
\hline 12 & 1582 & 593 & 112 & 43 & 2 & 1 & & & & & & \\
\hline 13 & 5715 & 2034 & 733 & 124 & 47 & 2 & 1 & & & & & \\
\hline 14 & 19428 & 7787 & 2550 & 889 & 136 & 51 & 2 & 1 & & & & \\
\hline 15 & 64557 & 27164 & 10371 & 3130 & 1061 & 148 & 55 & 2 & 1 & & & \\
\hline 16 & 211226 & 92661 & 37076 & 13531 & 3774 & 1249 & 160 & 59 & 2 & 1 & & \\
\hline 17 & 681895 & 309970 & 130205 & 49484 & 17331 & 4482 & 1453 & 172 & 63 & 2 & 1 & \\
\hline 18 & 2178208 & 1019459 & 445706 & 179365 & 64708 & 21835 & 5254 & 1673 & 184 & 67 & 2 & 1 \\
\hline
\end{tabular}


eliminated to save space and paper. Note that the table values are divided by 2 .

\section{SERIES ANALYSIS}

We assume that

$$
Q_{N}(\omega) \sim N^{\lambda(\omega)} \mu(\omega)^{N}
$$

by analogy with the RW and/or SAW of $\omega=0$, and form ratios of the alternate terms of $Q_{N}$ in order to reduce the marked odd-even oscillation characteristic of a loose-packed lattice:

$$
Q_{N} / Q_{N-2}=\left[1+2 \lambda / N+O\left(N^{-2}\right)\right] \mu^{2} .
$$

Then we construct Neville tables ${ }^{20}$ for the

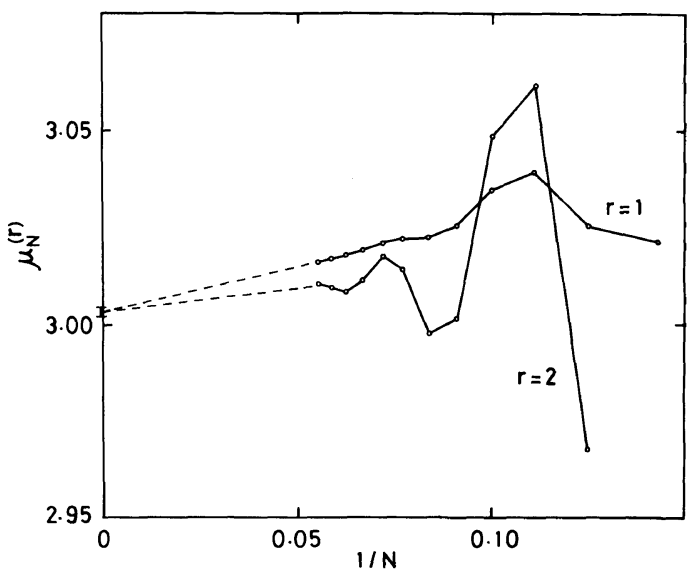

Figure 1. Estimation of $\mu$ from linear $(r=1)$ and quadratic $(r=2)$ extrapolants $\gamma_{N}^{(r)}$ for $\omega=0.45$ in a semiinfinite case.

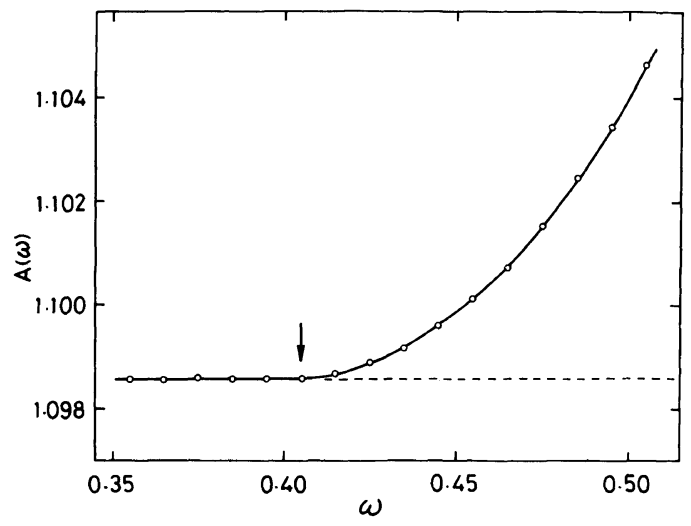

Figure 2. $A(\omega)$ as a function of $\omega$ for the semi-infinite system. The arrow indicates the transition point, $\omega_{\mathrm{c}}$. linear and quadratic extrapolants of $\mu_{N}=$ $\left(Q_{N} / Q_{N-2}\right)^{1 / 2}$ :

$$
\mu_{N}^{(\mathrm{r})}=\left[N \mu_{N}^{(\mathrm{r}-1)}-(N-2 r) \mu_{N-2}^{(\mathrm{r}-1)}\right] / 2 r
$$

for $r=1$ and 2 , with $\mu_{N}^{(0)} \equiv \mu_{N}$. We plot these extrapolants against $1 / N$ and estimate $\mu$ by extrapolating to $N \rightarrow \infty$ taking into account the curvature of convergence as a whole along with the damping oscillation around it. The last process is efficient when a confluent singularity exists. Thus we obtain A $(\omega)$ for given $\omega$ since $\log \mu=\lim _{N \rightarrow \infty} N^{-1} \log Q_{N}$ from eq 8 .

Figure 1 illustrates an example of the plot of linear $(r=1)$ and quadratic $(r=2)$ extrapolants $\mu_{N}^{(r)}$ against $1 / N$ which are calculated for $\omega=0.45$ from our data for the semi-infinite

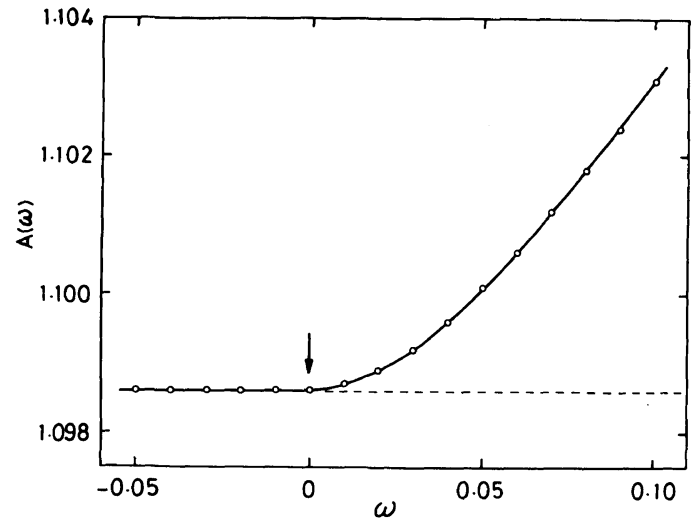

Figure 3. The same as Figure 2 but for the infinite system.

Table II. The values of $q_{\mathrm{i}} \equiv \log \Delta A_{\mathrm{i}} / \log \Delta \omega_{\mathrm{i}}(i=1-10)$ for the semi-infinite system; $\Delta A_{\mathrm{i}}=A\left(\omega_{\mathrm{i}}\right)-A_{0}$ and $\Delta \omega_{\mathrm{i}}=\omega_{\mathrm{i}}-\omega_{\mathrm{c}}$

\begin{tabular}{rccc}
\hline$i$ & $\Delta \omega_{\mathrm{i}}$ & $\Delta A_{\mathrm{i}}(\sim \pm 0.0002)$ & $q_{\mathrm{i}}$ \\
\hline 1 & 0.01 & 0.0001 & 2.000 \\
2 & 0.02 & 0.0003 & 2.074 \\
3 & 0.03 & 0.0006 & 2.116 \\
4 & 0.04 & 0.0010 & 2.146 \\
5 & 0.05 & 0.0015 & 2.171 \\
6 & 0.06 & 0.0020 & 2.209 \\
7 & 0.07 & 0.0026 & 2.238 \\
8 & 0.08 & 0.0032 & 2.274 \\
9 & 0.09 & 0.0038 & 2.314 \\
10 & 0.10 & 0.0045 & 2.347 \\
\hline
\end{tabular}


system; we estimate $\mu=3.003 \pm 0.001$. The values of $A(\omega)$ obtained for the semi-infinite system are plotted in Figure 2 as a function of $\omega$; the arrow indicates $\omega_{\mathrm{c}}=0.405$, which is in good agreement with the conjecture $\omega_{\mathrm{c}}=$ $\log (6 / 4)=0.4054 \ldots$, and it confirms $A=$ $A_{0}=\log 3$. for $\omega \leq \omega_{\mathrm{c}}$. The analogous plot for the infinite system is shown in Figure 3; it reproduces the expected value of $\omega_{c}=0$ and $A_{0}=\log 3$.

Equation 4 turns out to be

$$
q=\lim _{\Delta \omega \rightarrow+0} \frac{\log \Delta A}{\log \Delta \omega}
$$

where $\Delta A=A-A_{0}$ and $\Delta \omega=\omega-\omega_{\mathrm{c}}$. We can estimate q by calculating $\log \Delta A / \log \Delta \omega$ for different values of $\Delta \omega$ with an appropriate interval and extrapolating to $\Delta \omega \rightarrow 0$. The values of $\Delta A$ and $q_{i}=\log \Delta A_{i} / \log \Delta \omega_{i}$ for the semi-infinite system are given in Table II with the interval of $\Delta \omega_{i}-\Delta \omega_{i-1}=0.01$. The plot of $q_{i}$ against $\Delta \omega_{i}$ is shown in Figure 4; we estimate that $q=2.0_{-0.10}^{+0.15}$. The corresponding plot for the infinite system, which is shown in Figure 5, gives the estimate $q=2.0_{-0.08}^{+0.13}$. These estimates are in excellent agreement with the theoretical value of $q=2$.

We then estimate the exponent $\theta$ in eq 7 from the ratios

$$
\theta_{N}=N\left(1-f_{N} / f_{N-2}\right) / 2
$$

and using the method stated above. Figure 6 shows a plot of linear and quadratic extrapolants $\theta_{N}^{(\mathrm{r})}$ against $1 / N$ obtained for $\omega=$ 0.45 in a semi-infinite system; the value $\theta=$ $0.390 \pm 0.015$ was estimated. Figures 7 and 8 illustrate the plots of $\theta$ thus obtained as a function of $\omega$ for the semi-infinite and infinite systems, respectively. In both cases, $\theta$ decreases with increasing $\omega$, approaching unity for $\omega \ll \omega_{c}$ but still less than unity while it becomes negative for $\omega \gg \omega_{\mathrm{c}}$ contrary to expectation although the error bars enlarge there. We have the respective estimates $\theta=$ 0.52 and 0.51 at $\omega_{c}=0.405$ and 0 for the semiinfinite and infinite systems; these values are

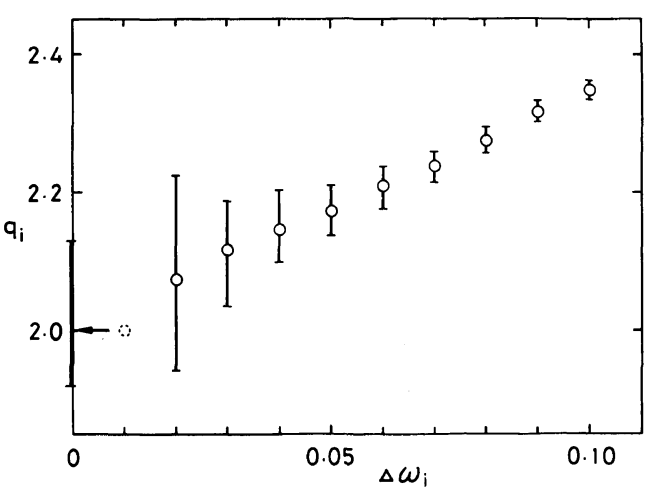

Figure 4. The plot of $q_{i} v s . \Delta \omega_{i}$ for the semi-infinite system. The arrow indicates the extrapolated value of $q$.

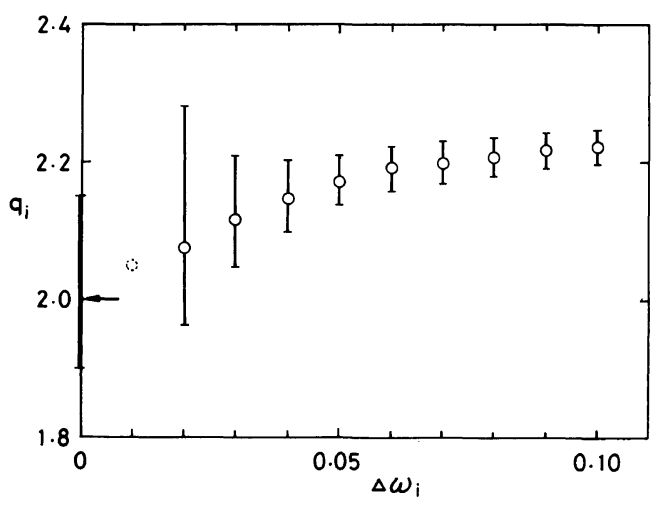

Figure 5. The same as Figure 4 but for the infinite system.

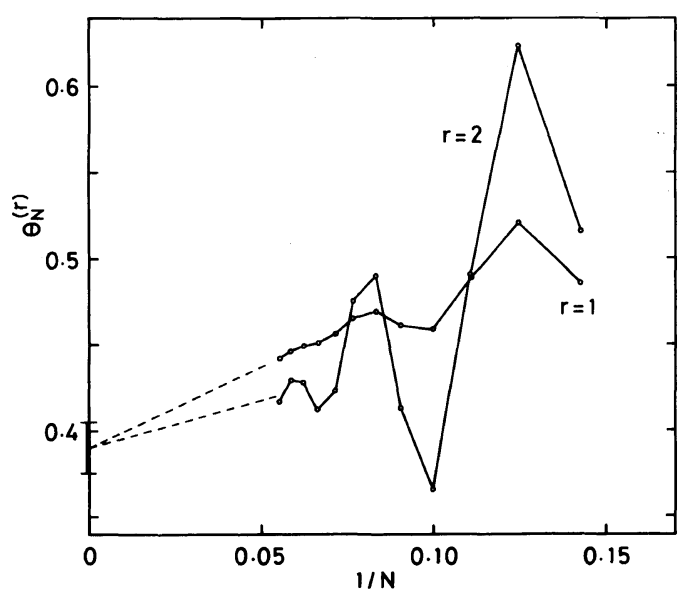

Figure 6. Estimation of $\theta$ from linear and quadratic extrapolants $\theta_{N}^{(r)}$ for $\omega=0.45$ in a semi-infinite case. 


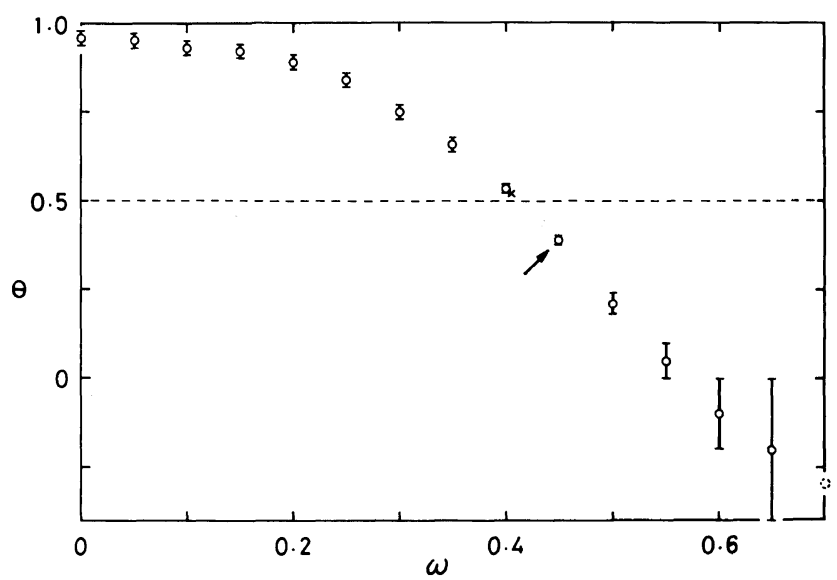

Figure 7. The plot of $\theta$ as a function of $\omega$ for the semi-infinite system. The arrow indicates an inflection point.

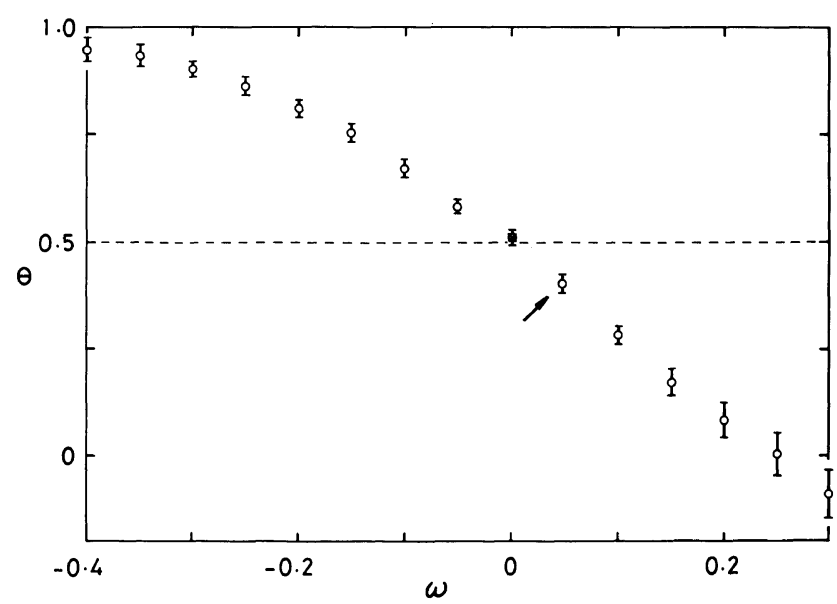

Figure 8. The same as Figure 7 but for the infinite system.

compatible with the theoretical result $\theta=$ 0.5 .

\section{DISCUSSION}

We have shown in the preceding section that our series analysis method is very reliable for the estimation of the transition point and critical exponents for the adsorption of a NRRW chain. In Figures 7 and 8, an inflection point is noted around the points indicated by arrows; $\theta=0.4$ is found at the inflection point in both plots. However, the point should not be regarded as a transition point since the values of $\omega$ and $\theta$ in this case are inconsistent with the expected values. A similar plot of $f_{N}$ in our previous paper $^{8}$ for SAWs in the infinite system gives $\theta=0.585$ (i.e., $\varphi=0.415$ ) for the choice of $\omega_{c}=0$ instead of the inflection point $\omega=0.20$. This value is inconsistent with the corresponding estimate $\varphi=0.40 \pm 0.02$ given by $\mathrm{Kremer}^{19}$ using a Monte Carlo method. Hence, for SAWs, we notice a discrepancy between values of respective $\varphi$ obtained through $q$ and $\theta$ although the reason is not clear. It suggests that the scaling theory for critical behavior at a defect plane deserves further examination, at least for 


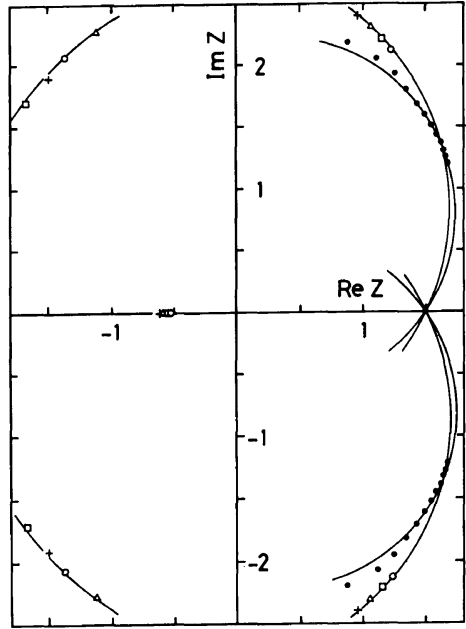

Figure 9. Location of the zeros of the partition function for semi-infinite system; $N=14(1), 15(+), 16(\triangle)$, $17(\square), 18(\bigcirc)$, and the largest real part (O) for $N=7-18$.

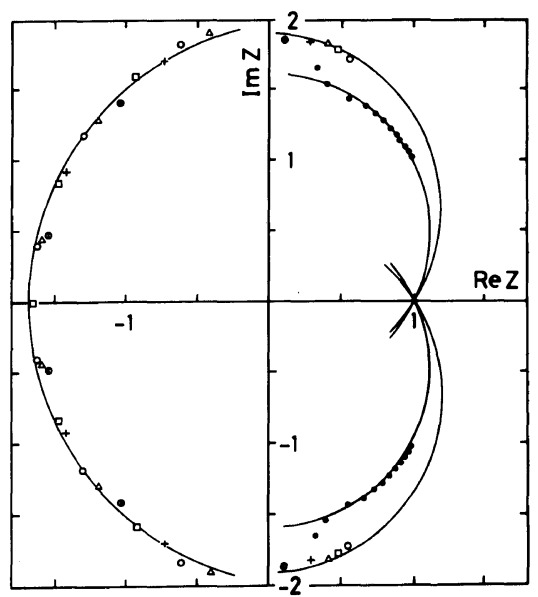

Figure 10. The same as Figure 9 but for the infinite system.

the $n=0$ model.

\section{Zeros of the Partition Function}

We rewrite eq 1 as

$$
Q_{N}(x)=\sum_{m} C_{N}(m) x^{m}
$$

where $x$ is real and positive. Equation 13 can be represented as

$$
Q_{N}(x)=C_{N}(1) \prod_{j=1}^{m_{N}}\left(1-x / z_{j}\right)
$$

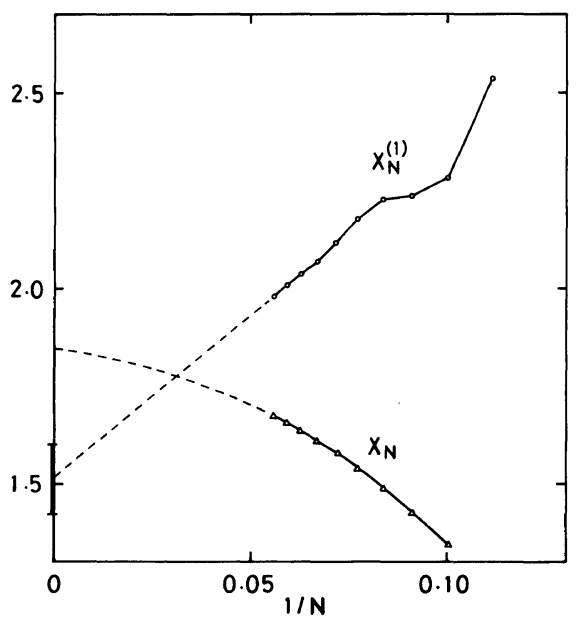

Figure 11. Plots of the real part $x_{N}$ of the zeros with largest real value and its linear extrapolant $x_{N}^{(1)}$ against $1 / N$ for the semi-infinite system.

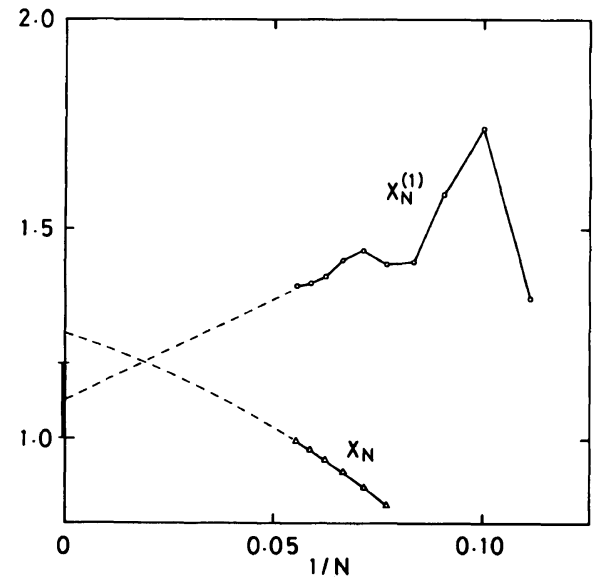

Figure 12. The same as Figure 11 but for the infinite system.

using the roots $z_{j}\left(j=1, \ldots, m_{N}\right)$ of $Q_{N}(z)=0$. None of the roots $z_{j}$ lies on the real positive axis of the complex $z$ plane since all coefficients $C_{N}(m)$ are positive. However, the zeros of $Q_{N}(z)$ must approach the positive real axis at $x=x_{\mathrm{c}}\left(=\mathrm{e}^{\omega} \omega_{\mathrm{c}}\right)$ as $N \rightarrow \infty$, seeing that $\lim _{N \rightarrow \infty} N^{-1} \log Q_{N}(x)$ is nonanalytic at $x=$ $x_{c}$ as expected from the Yang-Lee theory. ${ }^{23}$ Hence the location of the transition point can be estimated also from the zeros of the partition function, ${ }^{24}$ but the value seems ${ }^{11}$ 
always fairly larger than that estimated from the $A(\omega)$ vs. $\omega$ plot.

Figures 9 and 10 show the plots of the location of zeros close to the origin obtained for the semi-infinite and infinite systems, respectively. Black dots represent the zeros with the largest real part for $N=7-18$. The location of zeros is symmetric about the real axis but not the imaginary axis, and lies approximately on circles except for values on the negative real axis in the semi-infinite system. The circles for $\operatorname{Re} z>0$ cross at the point on the real axis. It is interesting to compare our results with the corresponding ones for the two-dimensional Ising model, ${ }^{25,26}$ where such zeros lie nearly on simple loci, often on circles.

In Figure 11, we plot the real part $x_{N}$ of the zeros with the largest real value in the semiinfinite system against $1 / N$ along with its linear extrapolant $x_{N}^{(1)}$. The latter gives $x_{c}=1.52$ \pm 0.08 , which coincides with the expected value $x_{c}=1.5$ and is in agreement with the crossing point on the real axis in Figure 9, while the former gives a much larger value. Similar plots for the infinite system are given in Figure 12. Almost the same situation is seen although the plot of $x_{N}^{(1)}$ yields $x_{\mathrm{c}}=1.09 \pm$ 0.09 a little larger than the theoretical value $x_{\mathrm{c}}=1$.

Acknowledgements. I am grateful to Mr. H. Ishikawa for assistance with computational work, and to Dr. K. Kremer for sending me his preprint prior to publication.

\section{REFERENCES}

1. M. N. Barber and B. W. Ninham, "Random and Restricted Walks," Gordon and Breach, New York,
1970, Chapter 11.

2. S. G. Whittington, Adv. Chem. Phys., 51, 1 (1982).

3. K. Binder and K. Kremer, "Scaling Phenomena in Disordered System, "Vol. 133, NATO ASI Series B, R. Pynn et al., Ed., Plenum, New York, N. Y., 1986.

4. P. G. de Gennes, J. Phys. (Paris), 37, 1445 (1976).

5. M. N. Barber, A. J. Guttmann, K. M. Middlemiss, G. M. Torrie, and S. G. Whittington, J. Phys. A, 11, 1833 (1978).

6. K. Binder, "Phase Transitions and Critical Phenomena," C. Domb and J. L. Lebowitz, Ed., Academic Press, New York, N. Y., 1983, Vol. 8, Chapter 1.

7. E. Eisenriegler, K. Kremer, and K. Binder, J. Chem. Phys., 77, 6296 (1982).

8. T. Ishinabe, J. Chem. Phys., 80, 1318 (1984).

9. A. J. Bray and M. A. Moore, J. Phys. A, 10, 1927 (1977).

1.0. H. Diehl and S. Dietrich, Phys. Rev. B, 24, 2878 (1981).

11. T. Ishinabe, J. Chem.Phys., 76, 5589 (1982); 77, 3171 (1982).

12. P. G. de Gennes, "Scaling Concepts in Polymer Physics," Cornell University, Ithaca, New York, 1979, Chapter 1.

13. K. Kremer, J. Phys. A, 16, 4333 (1983).

14. P. G. de Gennes and P. Pincus, J. Phys. Lett. (Paris), 44, 241 (1983).

15. P. G. de Gennes, Macromolecules, 14, 1637 (1981).

16. J. M. Hammersley, G. M. Torrie, and S. G. Whittington, J. Phys. A, 15, 539 (1982).

17. T. W. Burkhardt and E. Eisenriegler, Phys. Rev. B, 24, 1236 (1981).

18. H. Nakanishi, J. Phys. A, 14, L355 (1981).

19. K. Kremer, J. Chem. Phys., 83, 5882 (1985).

20. D. S. Gaunt and A. J. Guttmann, "Phase Transitions and Critical Phenomena," Vol. 3, C. Domb and M. S. Green, Ed., Academic Press, London, 1974, Chapter 4.

21. J. M. Hammersley, J. Appl. Prob. 19A, 327 (1982).

22. R. J. Rubin, J. Chem. Phys., 43, 2392 (1965).

23. C. N. Yang and T. D. Lee, Phys. Rev., 87, 404, 410 (1952).

24. R. Finsy, M. Janssens, and A. Bellemans, J. Phys. A, 8, L106 (1975).

25. S. Katsura, Prog. Theor. Phys., 38, 1415 (1967).

26. Y. Abe and S. Katsura, Prog. Theor. Phys., 43, 1402 (1970). 